\title{
Dynamic Path Planning of Unmanned Surface Vehicle Based on Genetic Algorithm with Sliding Curve Guidance System
}

\author{
Rusdhianto Effendi Abdul Kadir \\ Department of Electrical Engineering \\ Institut Teknologi Sepuluh Nopember \\ Surabaya, Indonesia \\ ditto@ee.its.ac.id
}

\author{
Mochammad Sahal \\ Department of Electrical Engineering \\ Institut Teknologi Sepuluh Nopember \\ Surabaya, Indonesia \\ sahal@ee.its.ac.id \\ Fian Ilham Pratama \\ Department of Electrical Engineering \\ Institut Teknologi Sepuluh Nopember \\ Surabaya, Indonesia \\ fianilham1@ee.its.ac.id
}

\author{
Nurlita Gamayanti \\ Department of Electrical Engineering \\ Institut Teknologi Sepuluh Nopember \\ Surabaya, Indonesia \\ lita@ee.its.ac.id
}

\begin{abstract}
Unmanned Surface Vehicle (USV) is an unmanned ship that is controlled through a remote control system (manual) or automatic control system (autopilot), move due to the thrust force from the thruster machine and can turning due to the deflection angle of rudder. The USV path planning system becomes an important task so that the ship can make the global trajectory with the minimum travel distance according to the desired navigation while at the same, able to avoid various obstacles from local dangerous situations that have the potential for collisions. To be able to do dynamic USV path planning, the Genetic Algorithm method with a sliding curve guidance system and PID MRAC controller is used. The use of this method gives smooth ship track performance with the shortest distance in a 400x400 square meter map with static and dynamic obstacles. In a dynamic environment, the path re-planning process that takes place in 0.98 seconds is able to find a new path that does not collide the obstacles. For the purposes of algorithm validation, the simulation is performed using MATLAB software with real ship parameters of 6 meters length USV.
\end{abstract}

Keywords-USV, genetic algorithm, PID MRAC, path planning, dynamic and static obstacle, sliding curve, guidance system

\section{INTRODUCTION}

Indonesia is a country that has a very large sea area, where $2 / 3$ of the territory is sea area. This makes Indonesia as one of the countries that has great potential in the marine sector. With a large sea area and all the maritime potential that is owned, the sea area that must be protected by the government is not small. Meanwhile, the problem faced by the Indonesian Navy as the agency authorized in maritime defense is the limited number of personnel, patrol boats, equipment, and funds. In this era of the rapid development of technology, human tasks can be replaced by the presence of robotic technology such as autonomous surface vessels. Surface vessel robot technology called Unmanned Surface Vehicle (USV) can replace the task of the Indonesian Navy in conducting automated patrols in Indonesia's sea territory, which means that can reduce the safety risk of the personnel on duty.

USV trajectory planning is an optimization complicated problem that must ensure the USV can navigate from initial location to goal location with a smooth path, minimum travel distance, and also able to avoid any possible collision obstacles in the dynamic marine environment. In recent decades, researchers have been searching alternative and more efficient ways to solve the path planning problems [1].

Different conventional approaches have been developed to solve path planning problems [2] such as cell decomposition, road map and potential field. Most of these approaches are based on the configuration space concept [3]. In addition to their lack of adaptively and robustness, conventional approaches are not suitable for dynamic environments because they utilize a sequential search algorithm to generate a single solution. This solution may become infeasible when a change in the environment is detected and a new solution has to be generated from scratch. Genetic Algorithms have been recognized as one of the most robust search techniques for complex and illbehaved objective functions [4].

Precise and smooth tracking of the trajectory during maneuvering is very important, especially for an autonomous surface vessel in the water environment $[5,6]$ with dynamic obstacles and natural disturbance such as sea current, waves, and wind. A guidance system is needed to guide the USV to follow a trajectory based on planned track information and target waypoints. The USV model used is a high-order and non-linear plant model with 6 Degree of Freedom, so a robust non-linear control system is needed to control the speed and steering (rudder angle) of the USV accurately according to the guidance system reference. In this paper, a dynamic path planning algorithm which includes global path planning and local path planning for USV based on genetic algorithm is proposed. Sliding curve guidance and discrete PID MRAC controller are used for the USV trajectory tracking system.

This paper is organized as follows. In section 2 , the mathematic model of USV is presented and USV parameter are listed. In section 3, the proposed path planning algorithm based on GA described in detail. In section 3, the sliding curve guidance strategy is presented, followed by the design of manuveur control system of USV using PIDMRAC in section 4. Section 5 presents the simulation of USV movement using Matlab in order to validate capability 
of genetic algorithm to perform obstacle avoidance. A conclusion of this paper is given in section 6 .

\section{Methods}

There are four points will be explained in the following below. A mathematical modeling of USV as a formula to simplify this dynamic path planning of USV, the path planning based on genetic algorithm presents a logic way to solve this simulation, a sliding curve guidance strategy as a method to complete this research, and maneuver control system of USV describes an adaptive control system to the autopilot system in USV.

\section{A. Mathematical Modeling of USV}

The USV used in this paper is 6 meters length USV using real ship parameters from the research results of the Systems and Cybernetics Laboratory of the ITS Electrical Engineering Department. The USV design is shown in Figure 1.

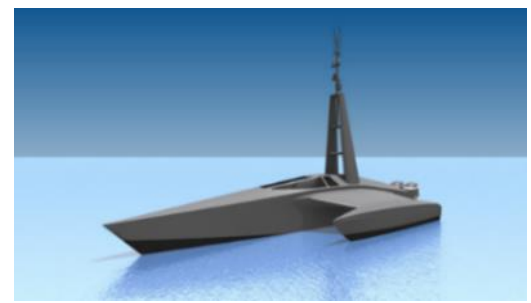

Fig. 1. Trimaran USV

The general motion of USV is expressed in terms of 6 degrees of freedom, as in Figure 2. The first three coordinates $(x, y, z)$ and their three first derivatives represent the general USV translation position and movement, while the last three coordinates $(\phi, \theta, \psi)$ and last three derivatives represent the general USV direction and movement of rotation.

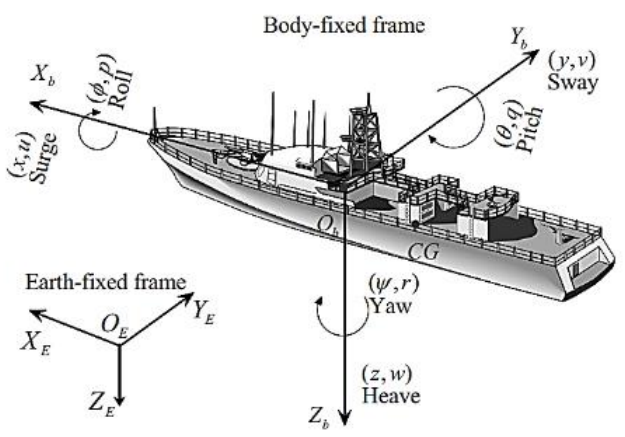

Fig. 2. The general USV movement

The general motion of USV variable can be represented by the following vectors,

$$
\begin{array}{lll}
\eta=\left[\begin{array}{ll}
\eta_{1} & \eta_{2}
\end{array}\right]^{T}, & \eta_{1}=\left[\begin{array}{lll}
x & y & z
\end{array}\right]^{T}, & \eta_{2}=\left[\begin{array}{lll}
\phi & \theta & \psi
\end{array}\right]^{T} \\
v=\left[\begin{array}{ll}
v_{1} & v_{2}
\end{array}\right]^{T}, & v_{1}=\left[\begin{array}{lll}
u & v & w
\end{array}\right]^{T}, & v_{2}=\left[\begin{array}{lll}
p & q & r
\end{array}\right]^{T} \\
\tau=\left[\begin{array}{ll}
\tau_{1} & \tau_{2}
\end{array}\right]^{T}, & \tau_{1}=\left[\begin{array}{lll}
X & Y & Z
\end{array}\right]^{T}, & \tau_{2}=\left[\begin{array}{lll}
K & M & N
\end{array}\right]^{T}
\end{array}
$$

Where,

$\eta$ : The position and orientation vector with coordinates in the earth-fixed frame

$v$ : The linear and angular velocity vector with coordinates in the body-fixed frame $\tau$ : The forces and moments acting on the vessel in the body-fixed frame

The USV mathematical model is given by,

$$
\begin{aligned}
& \dot{\eta}=\mathrm{J}(\eta) \mathrm{v} \\
& \mathrm{M} \dot{\mathrm{v}}=-\mathrm{C}(\mathrm{v}) \mathrm{v}-\mathrm{D}(\mathrm{v}) \mathrm{v}-\mathrm{g}(\eta)+\tau+\tau_{\mathrm{E}} \\
& \tau_{\mathrm{E}}=\tau_{\mathrm{E}}{ }^{\mathrm{cu}}+\tau_{\mathrm{E}}{ }^{\mathrm{wa}}+\tau_{\mathrm{E}}{ }^{\mathrm{wi}}
\end{aligned}
$$

In this paper, we assume that the center of gravity and the center of buoyancy are located vertically on the $O_{b} Z_{b^{-}}$ axis, and that there are no couplings (off-diagonal terms) in the matrices $M, D$, and $D_{n}(v)$ [7]. In this assumption, the general USV model in equation 1 can be simplified to the USV 6 Degrees of Freedom Model as follows,

$$
\begin{aligned}
& \dot{\eta_{1}}=\mathrm{J}_{1}\left(\eta_{2}\right) \mathrm{v}_{1} \\
& \mathrm{M}_{1} \dot{\mathrm{v}}_{1}=-\mathrm{C}_{1}\left(\mathrm{v}_{1}\right) \mathrm{v}_{2}-\mathrm{D}_{1} \mathrm{v}_{1}-\mathrm{D}_{\mathrm{n} 1}\left(\mathrm{v}_{1}\right) \mathrm{v}_{1}+\tau_{1}+\tau_{1 \mathrm{E}} \\
& \dot{\eta_{2}}=\mathrm{J}_{2}\left(\eta_{2}\right) \mathrm{v}_{2}
\end{aligned}
$$

$\mathrm{M}_{2} \dot{\mathrm{v}}_{2}=-\mathrm{C}_{1}\left(\mathrm{v}_{1}\right) \mathrm{v}_{1}-\mathrm{C}_{2}\left(\mathrm{v}_{2}\right) \mathrm{v}_{2}-\mathrm{D}_{2} \mathrm{v}_{2}-\mathrm{D}_{\mathrm{n} 2}\left(\mathrm{v}_{2}\right) \mathrm{v}_{2}-$ $\mathrm{g}_{2}\left(\eta_{2}\right)+\tau_{2}+\tau_{2 \mathrm{E}}$

$J_{1}\left(\eta_{2}\right)$ and $J_{1}\left(\eta_{2}\right)$ are a transformation matrix, which is related through the functions of the Euler angles: roll $(\phi)$, pitch $(\theta)$, and yaw $(\psi)$ defined as follows,

$$
\begin{aligned}
& J_{1}\left(\eta_{2}\right)= \\
& {[\cos (\psi) \cos (\theta)-\cos (\psi) \cos (\phi)+\sin (\phi) \sin (\theta) \cos (\psi)} \\
& \sin (\psi) \cos (\theta) \quad \cos (\psi) \cos (\phi)+\sin (\phi) \sin (\theta) \sin (\psi) \\
& -\sin (\theta) \quad \sin (\phi) \cos (\theta) \\
& \sin (\psi) \sin (\phi)+\sin (\theta) \cos (\psi) \cos (\phi) \\
& -\cos (\psi) \sin (\phi)+\sin (\theta) \sin (\psi) \cos (\phi) \\
& \cos (\phi) \cos (\theta) \\
& {\left[\begin{array}{ccc}
1 & \sin (\phi) \tan (\theta) & \cos (\phi) \tan (\psi) \\
0 & \cos (\phi) & -\sin (\phi) \\
0 & \sin (\phi) / \cos (\theta) & \cos (\phi) / \cos (\theta)
\end{array}\right]}
\end{aligned}
$$

$\mathrm{M}$ is a sum of the rigid body system inertia matrix and the added mass matrix defined as follows,

$$
\begin{aligned}
& M_{1}=\left[\begin{array}{ccc}
m_{11} & 0 & 0 \\
0 & m_{22} & 0 \\
0 & 0 & m_{33}
\end{array}\right] \\
& M_{2}=\left[\begin{array}{ccc}
m_{44} & 0 & 0 \\
0 & m_{55} & 0 \\
0 & 0 & m_{66}
\end{array}\right]
\end{aligned}
$$

With,

$$
\begin{aligned}
& \mathrm{m}_{11}=\mathrm{m}-\mathrm{X}_{\dot{\mathrm{u}}}, \mathrm{m}_{22}=\mathrm{m}-\mathrm{Y}_{\dot{\mathrm{v}}}, \\
& \mathrm{m}_{33}=\mathrm{m}-\mathrm{Z}_{\dot{\mathrm{w}}}, \mathrm{m}_{44}=\mathrm{I}_{\mathrm{x}}-\mathrm{K}_{\dot{\mathrm{p}}}, \\
& \mathrm{m}_{55}=\mathrm{I}_{\mathrm{y}}-\mathrm{M}_{\dot{\mathrm{q}}}, \mathrm{m}_{66}=\mathrm{I}_{\mathrm{z}}-\mathrm{N}_{\dot{\mathrm{r}}},
\end{aligned}
$$

$\mathrm{C}(\mathrm{v})$ is a sum of the rigid body Coriolis and centripetal matrix and the hydrodynamic Coriolis and centripetal matrix defined as follows,

$$
\begin{aligned}
& C_{1}\left(v_{1}\right)=\left[\begin{array}{ccc}
0 & m_{33} w & -m_{22} v \\
-m_{33} w & 0 & m_{11} u \\
m_{22} v & -m_{11} u & 0
\end{array}\right] \\
& C_{2}\left(v_{2}\right)=\left[\begin{array}{ccc}
0 & m_{66} r & -m_{55} q \\
-m_{66} r & 0 & m_{44} p \\
m_{55} q & -m_{44} p & 0
\end{array}\right]
\end{aligned}
$$


$\mathrm{D}$ and $\mathrm{D}_{\mathrm{n}}(\mathrm{v})$ each are the linear damping matrix and the nonlinear damping matrix defined as follows,

$$
\begin{aligned}
& D_{1}=\left[\begin{array}{ccc}
-X_{u} & 0 & 0 \\
0 & -Y_{v} & 0 \\
0 & 0 & -Z_{w}
\end{array}\right] \\
& D_{2}=\left[\begin{array}{ccc}
-K_{p} & 0 & 0 \\
0 & -M_{q} & 0 \\
0 & 0 & -N_{r}
\end{array}\right]
\end{aligned}
$$

$\mathrm{D}_{\mathrm{n} 1}\left(\mathrm{v}_{1}\right)=$

$$
\left[\begin{array}{ccc}
\sum_{i=2}^{3} d_{u i}|u|^{i-1} & 0 & 0 \\
0 & \sum_{i=2}^{3} d_{v i}|v|^{i-1} & 0 \\
0 & 0 & \sum_{i=2}^{3} d_{w i}|w|^{i-1}
\end{array}\right]
$$

$\mathrm{D}_{\mathrm{n} 2}\left(\mathrm{v}_{2}\right)=$

$$
\left[\begin{array}{ccc}
\sum_{i=2}^{3} d_{p i}|p|^{i-1} & 0 & 0 \\
0 & \sum_{i=2}^{3} d_{q i}|q|^{i-1} & 0 \\
0 & 0 & \sum_{i=2}^{3} d_{r i}|r|^{i-1}
\end{array}\right]
$$

Where $\mathrm{d}_{\mathrm{ui}}, \mathrm{d}_{\mathrm{vi}}, \mathrm{d}_{\mathrm{wi}}, \mathrm{d}_{\mathrm{pi}}, \mathrm{d}_{\mathrm{qi}}$, and $\mathrm{d}_{\mathrm{ri}}$ for $\mathrm{i}=2,3$ are the nonlinear hydrodynamic damping coefficients.

$$
g_{2}\left(\eta_{2}\right)=\left[\begin{array}{c}
\rho g \nabla \overline{G M_{T}} \sin (\phi) \cos (\theta) \\
\rho g \nabla \overline{G M_{L}} \sin (\theta) \\
0
\end{array}\right]
$$

Where $\rho, g, \nabla, G M_{T}$ and $G M_{L}$ are the density of the water, the acceleration due to gravity, the volume of displaced water, the transverse metacentric height, and the longitudinal metacentric height.

The propulsion force and moment vectors $\tau_{1}$ and $\tau_{2}$ are

$$
\tau_{1}=\left[\begin{array}{c}
\tau_{u} \\
0 \\
0
\end{array}\right], \quad \tau_{2}=\left[\begin{array}{c}
\tau_{p} \\
\tau_{q} \\
\tau_{r}
\end{array}\right]
$$

The environmental disturbance vectors $\tau_{I E}$ and $\tau_{2 E}$ are given by,

$$
\tau_{1 E}=\left[\begin{array}{c}
\tau_{E u} \\
\tau_{E v} \\
\tau_{E w}
\end{array}\right], \quad \tau_{2 E}=\left[\begin{array}{c}
\tau_{E p} \\
\tau_{E q} \\
\tau_{E r}
\end{array}\right]
$$

Where $\tau_{E u}, \tau_{E v}, \tau_{E w}, \tau_{E p}, \tau_{E q}$ dan $\tau_{E r}$ are the environment disturbances moments of forces acting on the axis of surge, sway, heave, roll, pitch, and yaw respectively. In this paper, only environmental disturbance forces acting on the surge $(x)$, sway $(y)$ and yaw $(\psi)$ are applied.

$$
\begin{aligned}
\boldsymbol{\tau}_{\boldsymbol{E}}^{\boldsymbol{w} \boldsymbol{i}} & =\frac{1}{2} \rho_{a} V_{w}^{2}\left[\begin{array}{c}
C_{x}\left(\gamma_{w}\right) A_{F w} \\
C_{y}\left(\gamma_{w}\right) A_{L w} \\
C_{N}\left(\gamma_{w}\right) A_{L w} L_{O A}
\end{array}\right] \\
\boldsymbol{\tau}_{\boldsymbol{E}}^{\boldsymbol{c u}} & =\frac{1}{2} \rho_{w} V_{c}^{2}\left[\begin{array}{c}
C_{x}\left(\gamma_{c}\right) A_{F c} \\
C_{y}\left(\gamma_{c}\right) A_{L c} \\
C_{N}\left(\gamma_{c}\right) A_{L c} L_{O A}
\end{array}\right] \\
\boldsymbol{\tau}_{\boldsymbol{E}}^{\boldsymbol{w a}} & =\frac{1}{2} \rho_{w}\left(A_{w a}\right)^{2} \sin (\omega t+\theta)\left[\begin{array}{c}
C_{x}\left(\gamma_{w a}\right) A_{F c} \\
C_{y}\left(\gamma_{w a}\right) A_{L c} \\
C_{N}\left(\gamma_{w a}\right) A_{L c} L_{O A}
\end{array}\right]
\end{aligned}
$$

where $\tau_{E}{ }^{w i}, \tau_{E}{ }^{c u}$ and $\tau_{E}{ }^{w a}$ are wind, current and wave induced forces in the surge, sway, yaw respectively, $\rho_{\mathrm{a}}$ is the air density, $\rho_{\mathrm{w}}$ is the water density, $V_{w}$ is the wind speed, $V_{c}$ is the current speed, $A_{w a}$ is wave amplitude, $A_{F w}$ is the frontal projected area, $A_{L w}$ is the lateral projected area, $A_{F c}$ is the submerged frontal projected area, $A_{L c}$ is the submerged

\begin{tabular}{|c|c|c|}
\hline Symbol & Description & Value \\
\hline 1 & Ship length & 6 \\
\hline $\mathrm{m}$ & Ship mass & 446,2810 \\
\hline$\rho_{\mathrm{w}}$ & Water density & 1025 \\
\hline$\rho_{\mathrm{a}}$ & Air density & 1.225 \\
\hline $\mathrm{I}_{\mathrm{X}}$ & Moments of inertia about $\mathrm{O}_{b} X_{b}$ & 459,6837 \\
\hline $\mathrm{I}_{\mathrm{Y}}$ & Moments of inertia about $\mathrm{O}_{b} \mathrm{Y}_{\mathrm{b}}$ & 27000 \\
\hline $\mathrm{I}_{\mathrm{Z}}$ & Moments of inertia about $\mathrm{O}_{\mathrm{b}} \mathrm{Z}_{\mathrm{b}}$ & 27000 \\
\hline $\mathrm{X}_{\dot{\mathrm{u}}}$ & $\begin{array}{l}\text { Added mass of ship in the direction of the } \mathrm{x} \\
\text { axis due to the ship's acceleration } \dot{\mathrm{u}}\end{array}$ & 133,8843 \\
\hline $\mathrm{Y}_{\dot{\mathrm{v}}}$ & $\begin{array}{l}\text { Added mass of ship in the direction of the y } \\
\text { axis due to the ship's acceleration } \dot{v}\end{array}$ & 89,2562 \\
\hline$Z_{\dot{\mathrm{w}}}$ & $\begin{array}{l}\text { Added mass of ship in the direction of the } \mathrm{z} \\
\text { axis due to the ship's acceleration } \dot{\mathrm{w}}\end{array}$ & 83,3058 \\
\hline $\mathrm{K}_{\dot{p}}$ & $\begin{array}{l}\text { Added mass of ship in the direction of the } \phi \\
\text { axis due to the ship's acceleration } \dot{p}\end{array}$ & 0 \\
\hline $\mathrm{M}_{\dot{\mathrm{q}}}$ & $\begin{array}{l}\text { Added mass of ship in the direction of the } \theta \\
\text { axis due to the ship's acceleration } \dot{q}\end{array}$ & 17400 \\
\hline $\mathrm{N}_{\dot{\mathrm{r}}}$ & $\begin{array}{l}\text { Added mass of ship in the direction of the } \\
\psi \text { axis due to the ship's acceleration } \dot{r}\end{array}$ & 17399 \\
\hline $\mathrm{X}_{\mathrm{u}}$ & $\begin{array}{l}\text { Linear damper constant } \mathrm{x} \text { due to translation } \\
\text { speed } \mathrm{u}\end{array}$ & 74,3802 \\
\hline $\mathrm{Y}_{\mathrm{v}}$ & $\begin{array}{l}\text { Linear damper constant y due to translation } \\
\text { speed } v\end{array}$ & 431,4050 \\
\hline $\mathrm{Z}_{\mathrm{w}}$ & $\begin{array}{l}\text { Linear damper constant } \mathrm{z} \text { due to translation } \\
\text { speed } \mathrm{w}\end{array}$ & -4314 \\
\hline $\mathrm{K}_{\mathrm{p}}$ & $\begin{array}{l}\text { Linear damper constant } \phi \text { due to translation } \\
\text { speed } p\end{array}$ & -23802 \\
\hline $\mathrm{M}_{\mathrm{q}}$ & $\begin{array}{l}\text { Linear damper constant } \theta \text { due to translation } \\
\text { speed } \mathrm{q}\end{array}$ & -446280 \\
\hline $\mathrm{N}_{\mathrm{r}}$ & $\begin{array}{l}\text { Linear damper constant } \psi \text { due to translation } \\
\text { speed } \mathrm{r}\end{array}$ & -23802 \\
\hline $\mathrm{d}_{\mathrm{u} 2} ; \mathrm{d}_{\mathrm{u} 3}$ & $\begin{array}{l}\text { Non-linear damper constant } \mathrm{x} \text { due to } \\
\text { translation speed } \mathrm{u}\end{array}$ & $\begin{array}{l}177,037 \\
0\end{array}$ \\
\hline$d_{v 2} ; d_{v 3}$ & $\begin{array}{l}\text { Non-linear damper constant y due to } \\
\text { translation speed } \mathrm{v}\end{array}$ & $\begin{array}{l}814,459 \\
0\end{array}$ \\
\hline $\begin{array}{c}\mathrm{d}_{\mathrm{w} 2} \\
\mathrm{~d}_{\mathrm{w} 3}\end{array}$ & $\begin{array}{l}\text { Non-linear damper constant } \mathrm{z} \text { due to } \\
\text { translation speed } \mathrm{w}\end{array}$ & $\begin{array}{c}814,459 \\
0\end{array}$ \\
\hline $\mathrm{d}_{\mathrm{p} 2} ; \mathrm{d}_{\mathrm{p} 3}$ & $\begin{array}{l}\text { Non-linear damper constant } \phi \text { due to } \\
\text { translation speed } \mathrm{p}\end{array}$ & $0 ; 0$ \\
\hline $\mathrm{d}_{\mathrm{q} 2} ; \mathrm{d}_{\mathrm{q} 3}$ & $\begin{array}{l}\text { Non-linear damper constant } \theta \text { due to } \\
\text { translation speed } \mathrm{q}\end{array}$ & $0 ; 0$ \\
\hline $\mathrm{d}_{\mathrm{r} 2} ; \mathrm{d}_{\mathrm{r} 3}$ & $\begin{array}{l}\text { Non-linear damper constant } \psi \text { due to } \\
\text { translation speed } r\end{array}$ & $\begin{array}{l}177,037 \\
0\end{array}$ \\
\hline$\overline{\overline{G M_{T}}}$ & Transverse metacentric height & 1,5 \\
\hline$\nabla$ & Volume of displaced water & 1,9698 \\
\hline$\overline{G M_{L}}$ & Longitudinal metacentric height & 10 \\
\hline$\frac{L}{\mathrm{~A}_{\mathrm{FW}}}$ & Frontal projected area & 2,0318 \\
\hline Symbol & Description & Value \\
\hline $\mathrm{A}_{\mathrm{Lw}}$ & Lateral projected area & 7,6067 \\
\hline$A_{F c}$ & Submerged frontal projected area & 0,3145 \\
\hline $\mathrm{A}_{\mathrm{Lc}}$ & Submerged lateral projected area & 2,1900 \\
\hline
\end{tabular}
lateral projected area, $L_{O A}$ is the overall length of ship, $\gamma$ is the relative angle that blew the ship described by equation $27, \beta$ is the direction of disturbance and $C_{x}, C_{y}, C_{N}$ are the coefficients in axis $\left[\begin{array}{lll}x & y & \psi\end{array}\right]^{\mathrm{T}}$.

$$
\gamma=\psi+\beta-\pi
$$

The Internal parameters of Trimaran USV described by Table 1.

TABLE I. USV PARAMETERS 


\section{B. Path Planning Based on Genetic Algorithm}

1) Concept of Path Planning

Path planning is an important task in USV navigation. Path planning allows USV to find the optimal path from the starting point to the endpoint, based on several performance indicators such as the minimum cost of travel, the shortest travel time, the shortest route. And during this process, USV needs to avoid obstacles that could be potential for collision. According to the type of environmental information used to calculate the optimal path, these path problems can be divided into two types: global path planning and local path planning [8]. The illustration of both path planning methods can be shown in Figure 3.

Global path planning aims to find an optimal path based on a global geographic map in a static environment. Global path planning is not sufficient to control a USV in realtime, especially when there are unexpected obstacles that appear during the travel (dynamic obstacles) because no sensors are used. To solve global path planning problems, information is needed in the form of a map containing information about the size and position of static obstacles, starting and end waypoint.

Local path planning is based on local environmental information and USV's state estimate and aims to plan local path without collisions in the dynamic environment. In this case, the path planning algoritam is required to be adaptive to the dynamic characteristics of the environment, by obtaining information (such as size, shape, and location) about the unknown obstacles in the local map through various USV sensors. When the USV sensor detects any dynamic obstacle that has the potential for collision, with local planning, USV is able to plan a new collision-free path.

Dynamic path planning is a combination of global path planning and local path planning where the global path between starting and endpoint will be updated through path re-planning if there are any obstacles detected in the local map or USV sensor range.

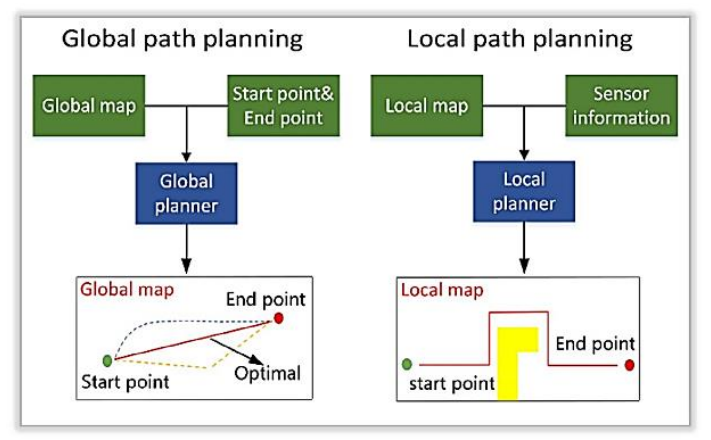

Fig. 3. Global and local path planning [8]

\section{2) Genetic Algorithm}

The genetic algorithm as a branch of the evolutionary algorithm is a heuristic search technique that can be used to find a solution (value) in a complex optimization problem. This algorithm is modeled based on Charles Darwin's theory of genetic processes that exist in living things, with the development of generations in a natural population, gradually following the principle of natural selection in which the best individuals in a population will survive. The fitness evaluation function plays an important role in genetic algorithms because it determines the quality of an individual.

The process of the genetic algorithm are as follows: first generates an initial population randomly, then the population is evaluated with a predetermined fitness function, then the population is processed (recombined) using genetic operators such as selection, crossover, and mutation based on a probability. thus generates new populations for the next generation. The process are repeated until they reach certain criteria. These criteria can be either the generation limit or the desired optimal value.

The number of chromosome length selections and the number of populations affect the number of iterations of the genetic algorithm, which will have an impact on the computation time of the genetic algorithm. In the real implementation of the use of genetic algorithms in the case of finding the optimal solution or minimum cost, the algorithm calculation time to find the individual according to the optimal solution is an indicator that must be minimized. The GA parameters used in dynamic path planning are shown in Table 2.

\section{TABLE II. GA PARAMETERS}

\begin{tabular}{|l|l|}
\hline \multicolumn{1}{|c|}{ Parameters } & \multicolumn{1}{c|}{ Type/Value } \\
\hline GA Type & Simple GA \\
\hline Selection Type & Roulette \\
\hline Crossover Type & Single point \\
\hline Mutation Type & Uniform \\
\hline $\begin{array}{l}\text { Maximum generation } \\
\text { (Global Path Planning) }\end{array}$ & 30 generation \\
\hline Maximum generation (Local Path Planning) & 20 generation \\
\hline Population Size & 20 individuals \\
\hline Chromosom Size & 4 bits (4 waypoint) \\
\hline Crossover Probability & 0,9 \\
\hline Mutation Probability & 0,35 \\
\hline
\end{tabular}

In this paper, the type of individual representation (chromosome) used in genetic algorithms is permutation coding. By presenting waypoints on a path with integers number: 1, 2, 3, ..., $\mathrm{n}$ as chromosomes. Each code/number from a waypoint can be regarded as a gene on a chromosome so that the chromosome is a string of codes from the waypoints that form a path and represent a sequence from starting point to the endpoint. In this path planning problem, a two-dimensional map at the xy coordinate is used to describe the optimal path based on the $\mathrm{x}$-axis chromosome and $\mathrm{Y}$-axis chromosome. Chromosome representation can be seen in Figure 4.

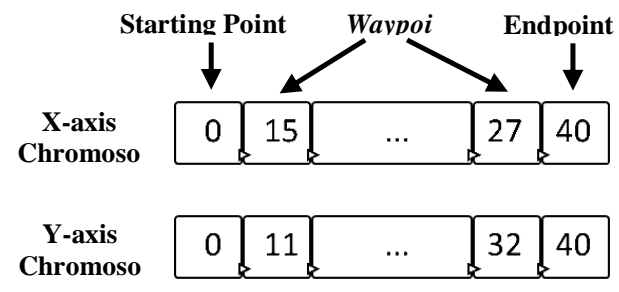

Fig. 4. GA Chromosome Representation

In this paper, the path planning algorithm is used to find the optimal path with the shortest distance from the starting point $(0,0)$ and the endpoint $(40,40)$ in a $40 \times 40$ cell global map in which 1 cell size represents 10 meters length. Thus, the fitness value of genetic algorithm is $1 /$ total distance 
(one per total distance). In this case, what is meant by total distance is the total distance calculated from the starting waypoint to endpoint. In global path planning, the starting point is the coordinate point of $(0,0)$, but in local path planning, the starting point is the coordinate of USV's location at the time when any potential collision obstacles are detected by USV. The equation of fitness function is given by,

$$
\begin{aligned}
& \operatorname{distance}(i, j)=\sqrt{\left(x_{i}-x_{j}\right)^{2}+\left(y_{i}-y_{j}\right)^{2}} \\
& \text { fitness function }=1 / \operatorname{distance}(i, j)
\end{aligned}
$$

Selection is used to determine the individuals who will play a role in forming a new population. The purpose of selection is to ensure that the individuals selected to follow the reproduction process have a high fitness value. The type of selection used in this research is roulette wheel selection. It can also be called a fitness proportionate selection, where the selection process is performed randomly, but chromosomes with a high fitness value are given a large probability (proportional).

An effort to maintain best chromosomes in a generation is by applying elitism. In elitism process, the chromosomes that have the highest fitness value will not follow the crossover and mutation process, but will be direct to be the next chromosome in new population. In this paper, elitism is applied by only taking the 1 best chromosome in order for the other population could have the opportunity to find a better solution.

Crossover is a process of crossing a pair of parent chromosomes to produce children (offspring) who will become individuals or new chromosomes in the population in the next generation. The number of chromosomes that are crossovered is determined by the crossover probability parameter $(\mathrm{Pc})$. The single point crossover can be shown in Figure 5.

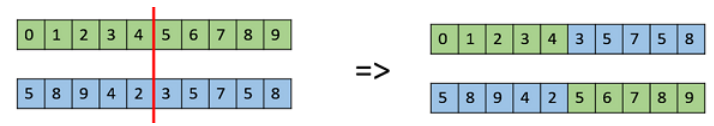

Fig. 5. Single point crossover [9]

Mutation is a genetic process to maintain the genetic diversity of a population. This is performend to prevent the population from trapping in the local optimal solution. In this paper, a uniform mutation type is used where the value of the selected gene is replaced with a uniform random value between the specified upper and lower bounds (coordinate points in 40x40 cell map). The uniform mutation is shown in Figure 6.

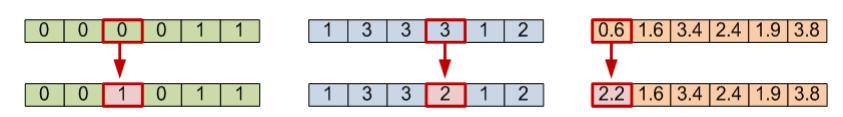

Fig. 6. Uniform mutation [9]

\section{3) Dynamic Path Planning}

To solve the path planning problems discussed in this paper, dynamic path planning based on the genetic algorithm will be used by combining global path planning and local path planning methods so that USV can avoid static and dynamic obstacles.

The trajectory tracking system is needed to show the algorithm's capability to replan a path that is suddenly blocked by an unpredicted and dynamic obstacle on the way to the endpoint based on USV's original (global) path on the global map. Dynamic path planning algorithm is given by:

1. Run a global path planning program based on the workflow of static path planning on a global map to obtain the set of waypoints that produce the shortest path between the start and end waypoints.

2. During the global path planning, if there is an obstacle detected within the range of USV's unsafe which indicates a potential collision will occur so that a path re-planning is needed.

3. Run a local path planning program based on the workflow of static path planning by using the last position of USV since the obstacle is detected as the first population of genetic algorithm to accelerate the algorithm process.

The path diagram for the path planning strategy in a dynamic environment is shown in Figure 7.

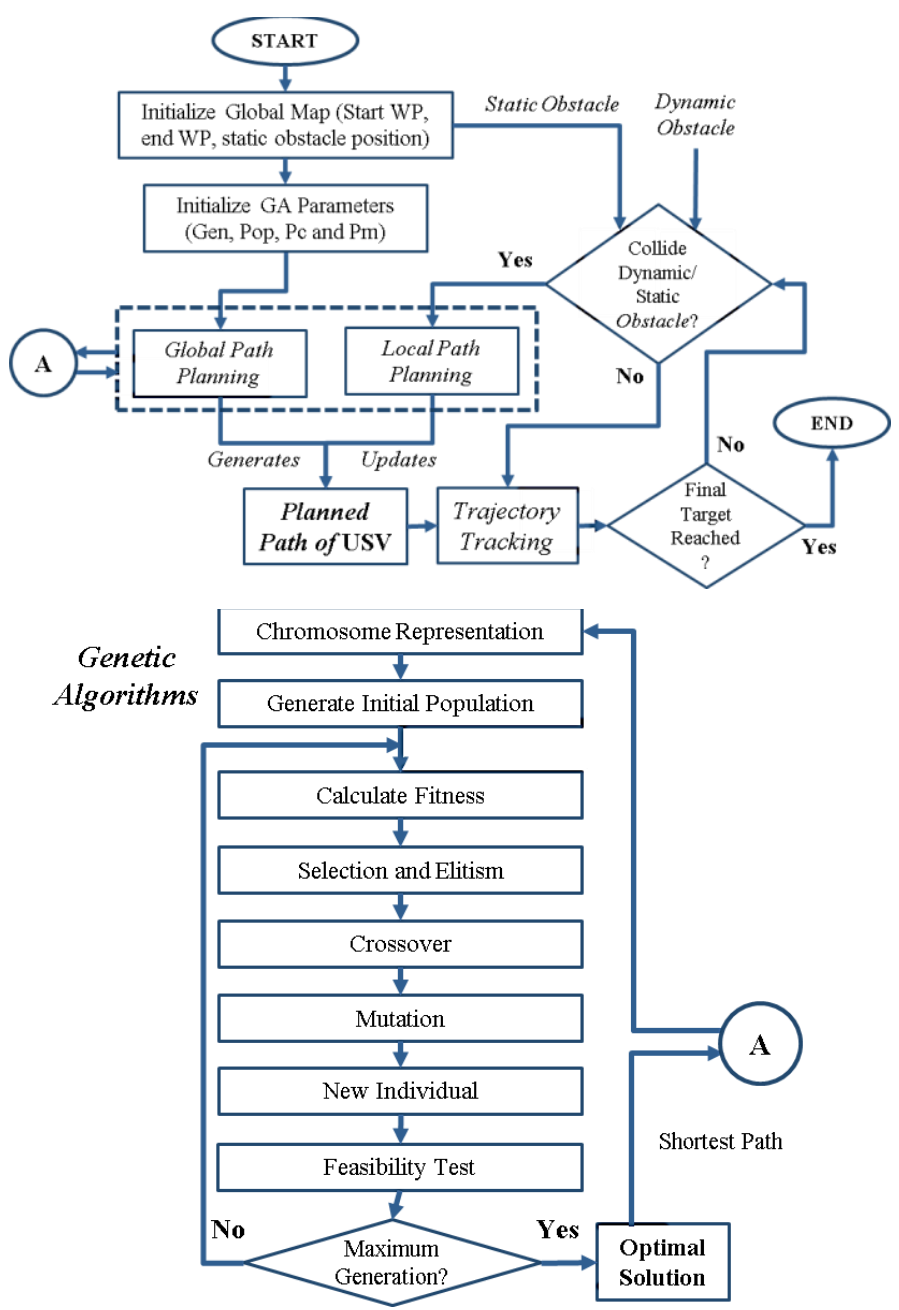

Fig. 7. The flow chart of dynamic path planning

The overall USV navigation system in dynamic path planning can be illustrated in Figure 8, first, initialize a global map containing information of the starting point and destination (goal), then global path planning is executed to produce a set of waypoints that form a collision-free optimal path between both of these points. The waypoint set is sent to the sliding curve guidance system block to obtain the target (reference) yaw angle which will be processed to the 
Discrete PID MRAC controller. Control commands are sent to the USV dynamics block to generate the output of yaw angle and translation speed according to the PID MRAC input reference. These two values will give the USV position in xy coordinate. During the process, if a dynamic obstacle is detected in the $+100 \mathrm{~m}$ unsafe range and collision is predicted to occur, local path planning is executed to obtain a new collision-free path and update the global route on the global map.

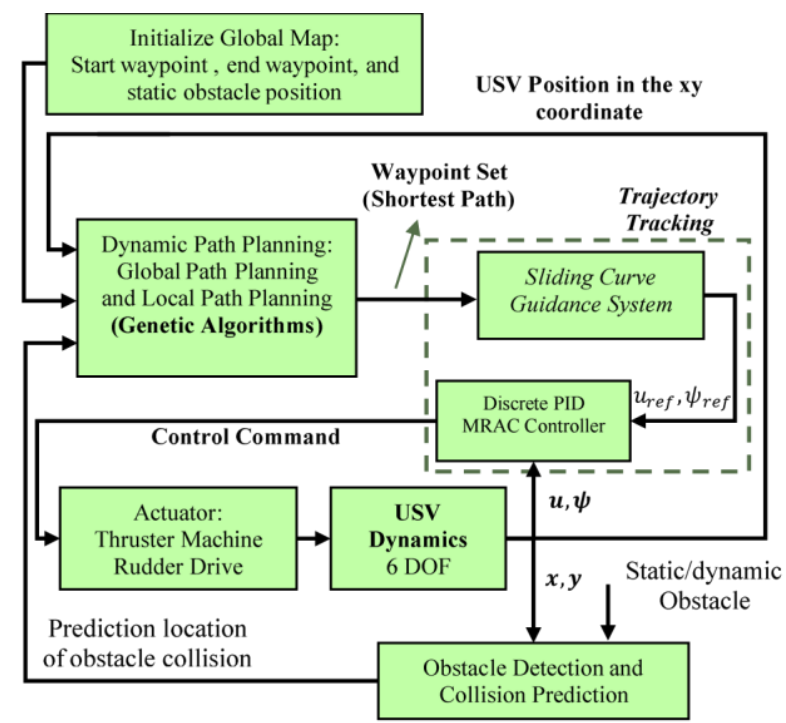

Fig. 8. USV navigation block diagram

\section{Sliding Curve Guidance Strategy}

After a collision-free trajectory has been created in the path planning design, the USV requires a guidance system to be able to follow the each waypoints according to the target trajectory. The USV guidance will receive information of the current USV position and the target waypoint. The information then will be processed into a calculation of the target USV heading angle that must be achieved so that the USV can move following the desired waypoint according to path planning based on genetic algorithms. The proposed guidance system method in USV simulation is the sliding curve guidance.

The sliding curve method is a trajectory tracking method that utilizes information on the heading of the ship and the target virtual moving along the sliding curve path so that it can be used to guide the USV movement to follow the target trajectory. The main objective of this sliding curve guidance method is to minimize the cross-track error or the difference in the distance between the USV position at any time against the existing trajectory and to equalize the heading angle of USV with the path angle. With a minimum cross-track error and the same direction of heading angle USV to the trajectory, the USV movement following the trajectory will be obtained. This sliding curve method was developed from the carrot-chasing method [10]. The sliding curve guiding algorithm for straight line trajectories is shown in Figure 2.4 .

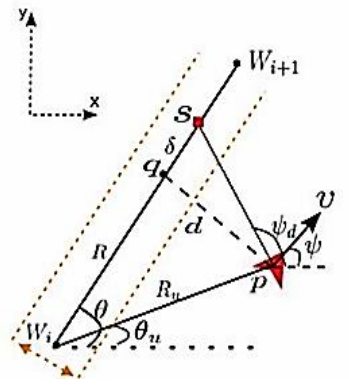

Fig. 9. Sliding curve guidance [10]

Based on Figure 9, $p$ is the coordinate of USV position on the $(x, y)$ axis, $\psi$ is the heading angle or direction towards USV, $d$ is the cross-track error, $W_{i}$ is the waypoint at the moment $i, W_{i+1}$ is the waypoint when $i+1, q$ is the point perpendicular from the USV to trajectory, $b$ is the width of the sliding curve, $s$ is the target virtual, and $\delta$ is the distance parameter that defines closed or far distance of the point $s$. The sliding curve method moves the target virtual point from one waypoint to the next, so that the path to be followed is an arrangement of points along the trajectory. The sliding curve algorithm is shown in Table 3.

TABLE III. SLIDING CURVE GuIDANCE ALGORITHM

\begin{tabular}{|c|l|l|}
\hline No. & \multicolumn{1}{|c|}{ Algorithm } & \multicolumn{1}{c|}{ Description } \\
\hline 1. & $\mathrm{~W}_{\mathrm{i}}=\left(\mathrm{x}_{\mathrm{i}}, \mathrm{y}_{\mathrm{i}}\right)$ & Waypoint at $\mathrm{i}$ \\
\hline 2. & $\mathrm{~W}_{\mathrm{i}+1}=\left(\mathrm{x}_{\mathrm{i}+1}, \mathrm{y}_{\mathrm{i}+1}\right)$ & Waypoint at $\mathrm{i}+2$ \\
\hline 3. & $\mathrm{p}=\left(\mathrm{x}_{\mathrm{i}}, \mathrm{y}_{\mathrm{i}}\right), \psi$ & $\begin{array}{l}\text { Position and orientation of } \\
\text { USV in the } x y \text { plane }\end{array}$ \\
\hline 4. & $\mathrm{R}_{\mathrm{u}}=\left\|\mathrm{W}_{\mathrm{i}}-\mathrm{p}\right\|$ & Distance $\mathrm{W}_{\mathrm{i}}$ to $\mathrm{p}$ \\
\hline 5. & $\theta=\operatorname{atan} 2\left(\mathrm{y}_{\mathrm{i}+1}-\mathrm{y}_{\mathrm{i}}, \mathrm{x}_{\mathrm{i}+1}-\mathrm{x}_{\mathrm{i}}\right)$ & $\theta$ in the $x y$ plane \\
\hline 6. & $\theta_{\mathrm{u}}=\operatorname{atan} 2\left(\mathrm{y}-\mathrm{y}_{\mathrm{i}}, \mathrm{x}-\mathrm{x}_{\mathrm{i}}\right)$ & $\theta_{\mathrm{u}}$ in the $x y$ plane \\
\hline 7. & $\beta=\theta-\theta_{\mathrm{u}}$ & $\beta$ in the $x y$ plane \\
\hline 8. & $R=\sqrt{R_{u}^{2}-\left(R_{u} \sin (\beta)\right)^{2}}$ & $\mathrm{R}$ in the $x y$ plane \\
\hline 9. & $\begin{array}{l}\mathrm{x}_{\mathrm{t}}{ }^{\prime} \leftarrow(\mathrm{R}+\delta) \cos \theta \\
\mathrm{R}+\delta) \sin \theta\end{array}$ & $\begin{array}{l}\text { The virtual target position }(s) \\
\text { on the path where dengan } \delta \text { is } \\
\text { the virtual target distance to } \\
\text { USV }\end{array}$ \\
\hline 10. & $\psi_{\mathrm{d}}=\operatorname{atan} 2\left(\mathrm{y}_{\mathrm{t}}{ }^{\prime}-\mathrm{y}, \mathrm{x}_{\mathrm{t}}{ }^{\prime}-\mathrm{x}\right)$ & Target heading angle $\left(\psi_{\mathrm{ref}}\right)$ \\
\hline
\end{tabular}

\section{Maneuver Control System Of USV}

\section{1) PID MRAC MIT Rule}

The autopilot system is developed so that USV is able to work at the speed and heading angle that suits the user by controlling the USV variables such as rudder angle and engine \%throttle. PID MRAC is a reference model based adaptive control system. Where to adjust the plant behavior of non-linear USV, a reference model is required. In PID MRAC controller as adaptive controller, the value of proportional gain $\left(\boldsymbol{K}_{\boldsymbol{p}}\right)$, derivative gain $\left(\boldsymbol{K}_{\boldsymbol{d}}\right)$ and integral gain $\left(\boldsymbol{K}_{i}\right)$ are automatically updated in such a way that plant follows the reference model [11]. The general PID MRAC block diagram can be seen in Figure 9.

In this paper, MIT rule is used for adjustment mechanism. According to gradient approach, a cost function $\psi(\phi)$ is decided in terms of tracking error $(e)$. The tracking error is defined as error between output of reference model and output of plant as follows,

$$
e_{u}=u(t)-y_{m}(t)
$$




$$
\psi(\phi)=\frac{1}{2} e_{u}(\phi)
$$

According to the MIT rule, the rate of change $\Phi$ is directly proportional to the negative gradient of the cost function, as shown in the following equation,

$$
\frac{\mathrm{d} \phi}{\mathrm{dt}}=-\gamma \frac{\partial \psi}{\partial \phi}
$$

Where $\Phi=$ controller parameter vector, $\mathrm{e}_{\mathrm{u}}=$ tracking error of $\mathrm{u}, \gamma=$ Adaptive Gain dan $\vartheta \psi / \vartheta \Phi=$ sensitivity derivative.

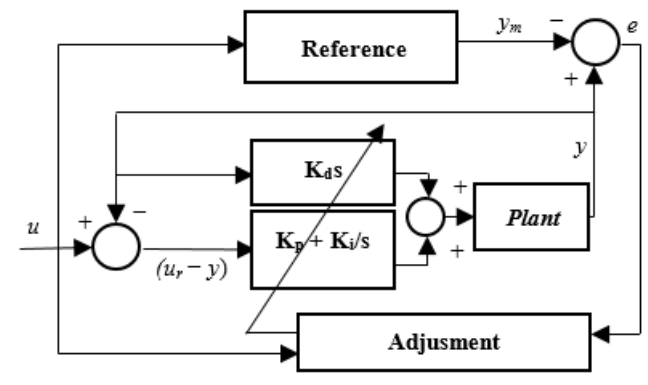

Fig. 10. PID MRAC block diagram

Based on Figure 10, the control signal (plant input) in the PID MRAC is defined as follows:

Where,

$$
u(s)=K_{P}\left[u_{r}-y_{m}\right]+\frac{K_{I}}{s}\left[u_{r}-y_{m}\right]+K_{D} s\left[u_{r}-y_{m}\right]
$$

$\mathrm{u}(\mathrm{s}) \quad=$ Control Signal

$\mathrm{y}_{\mathrm{m}} \quad=$ Reference Model Signal

$\mathrm{u}_{\mathrm{r}} \quad=$ Setpoint

$\mathrm{K}_{\mathrm{P}}, \mathrm{K}_{\mathrm{D}}, \mathrm{K}_{\mathrm{I}} \quad=$ PID MRAC Parameters

2) Maneuver Control System Using PID-MRAC

In this study, the USV autopilot control system was divided into 2 parts. The first part is a translation speed control system in the direction of the $\mathrm{x}$ axis $(\mathrm{u})$, where the PID MRAC controller with MIT Rule regulates the amount of thruster motor force $(\tau)$ or \%engine throttle. In the autopilot of translation speed, the PI controller is used. The second part is the heading angle adjustment system (pengaturan), where the PID MRAC controller with MIT Rule regulates the amount of propeller thruster turning angle that must be generated. In the autopilot of heading angle, a PD controller is used. The block diagram of the USV maneuver control system can be seen in Figure 11.

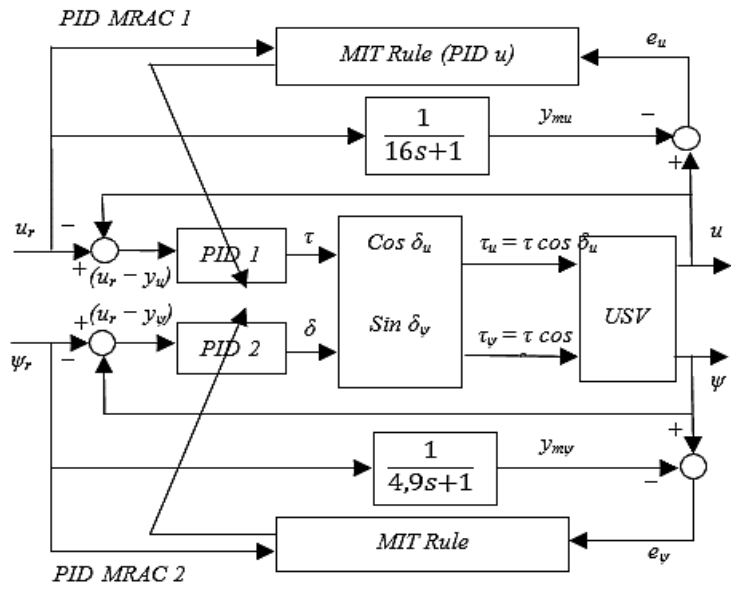

Fig. 11. Manuever control system of USV
The reference model used in the design of the $\mathrm{x}$-axis linear velocity control system and heading angle (yaw) respectively are:

$$
\begin{aligned}
& y_{m}=\frac{1}{16 s+1} \\
& y_{m}=\frac{1}{4,9 s+1}
\end{aligned}
$$

\section{RESULTS AND ANALYSIS}

The adaptive gain value $\gamma$ PID MRAC used for translation speed autopilot and USV heading angle is shown in Table 4.1 where PID MRAC 1 (translation speed autopilot) uses a PI controller and PID MRAC 2 (heading angle autopilot) uses a PD controller. In this simulation, disturbance was applied such as wind, current, and wave. The magnitude of wind, current, and wave are $0.1,0.1$, and 0.15 respectively.

\section{TABLE IV. PID MRAC PARAMETER}

\begin{tabular}{|c|c|c|}
\hline Adaptive Gain & PID MRAC 1 & PID MRAC 2 \\
\hline$\gamma_{P}$ (Adaptive Gain P) & 60 & 100 \\
\hline$\gamma_{\mathrm{I}}$ (Adaptive Gain I) & 1 & 0 \\
\hline$\gamma_{D}$ (Adaptive Gain D) & 0 & 1400 \\
\hline
\end{tabular}

Initially, a global map with a size of 40x40 was designed where the first waypoint is at the coordinate $(0,0)$ and the destination waypoint is at the coordinate $(40,40)$. The size of 1 grid on the global map represents an actual distance of 10 meters. After that, the genetic algorithm parameters in Table 2 are used and apply elitism. The simulation obstacle configuration can be seen in Table 5 .

TABLE V. OBSTACLE CONFIGURATION

\begin{tabular}{|c|c|c|}
\hline \multirow{2}{*}{ Obstacle } & \multicolumn{2}{|c|}{ Obstacle Configuration } \\
\cline { 2 - 3 } & Position & Velocity Vector \\
\hline \multirow{3}{*}{ Static } & $(10,17)$ & $0 \mathrm{~m} / \mathrm{s}$ \\
\cline { 2 - 3 } & $(26,26)$ & $0 \mathrm{~m} / \mathrm{s}$ \\
\cline { 2 - 3 } & Radius/edge length $=30 \mathrm{~m}$ \\
\hline \multirow{3}{*}{ Dynamic } & $(30,0)$ & $4,5 \mathrm{~m} / \mathrm{s}, 154,8^{\circ}$ \\
\cline { 2 - 3 } & $(20,36)$ & $3 \mathrm{~m} / \mathrm{s},-90^{\circ}$ \\
\cline { 2 - 3 } & \multicolumn{2}{|c|}{$(40,20)$} \\
\hline
\end{tabular}

USV simulations in dynamic environments are performed, to test the capabilities of the path planning algorithm based on genetic algorithms to avoid dynamic obstacles. In the obstacle configuration given by Table 5 , two static obstacles three ship-shaped moving obstacles are used with specific parameters size and velocity vectors. The simulation step figures are shown in Figure 12a-d and Figure 12e-f.

The path planning algorithm is run with MATLAB R2018b software and run on a computer with specifications: Intel Core i5 $2.60 \mathrm{GHz}$ processor, $4 \mathrm{~GB}$ RAM, and Windows 10 64-bit operating system.

The symbol and color codes in the simulation image are defined as follows: Waypoints are represented as small green circles, the static obstacles are represented as a red circle or red box with a certain size, the dynamic obstacles are represented as a red ship-shaped object, USV is represented as a blue ship-shaped object, the magenta curve represents the target trajectory of the path planning results, 
the blue curve represents the path of USV has reached, the red curve represents the dynamic obstacle trajectory.

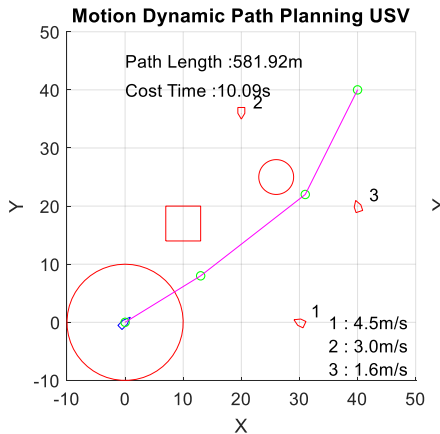

(a) $t=0$ second

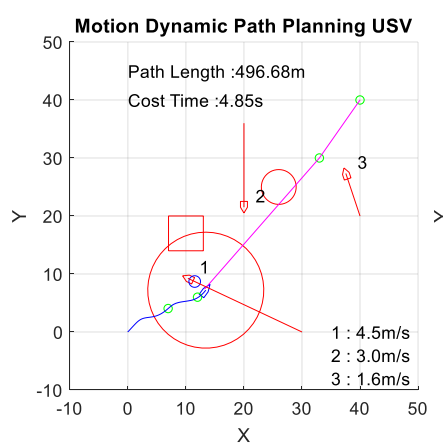

(c) $\mathrm{t}=48$ second

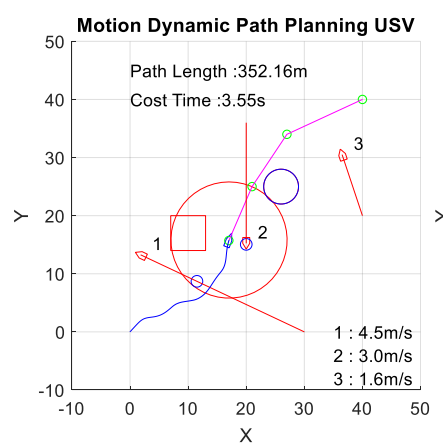

(e) $t=69$ second

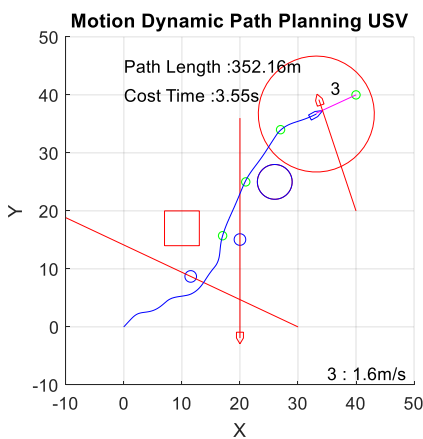

(g) $\mathrm{t}=126$ second

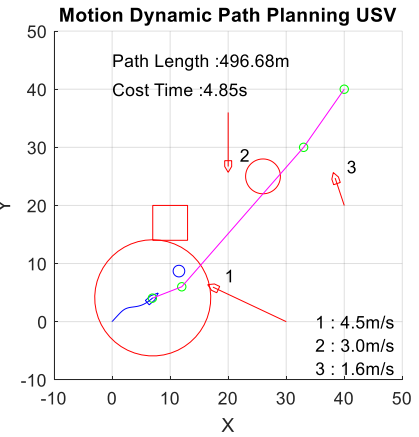

(b) $\mathrm{t}=30$ second

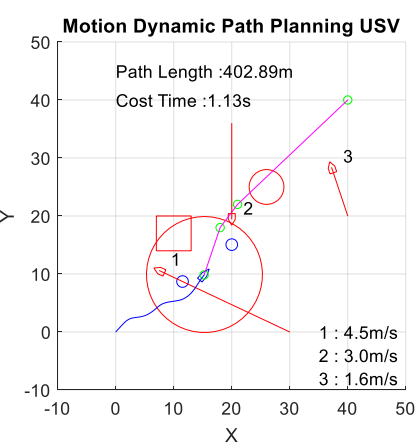

(d) $\mathrm{t}=56$ second

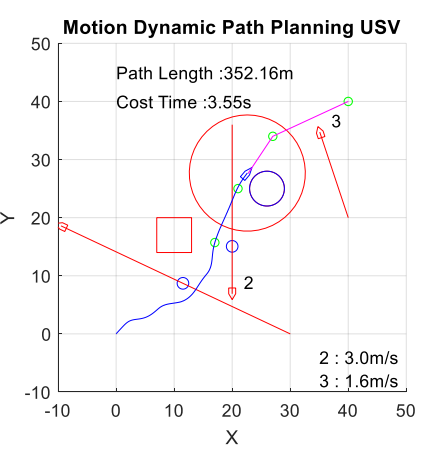

(f) $\mathrm{t}=97$ second

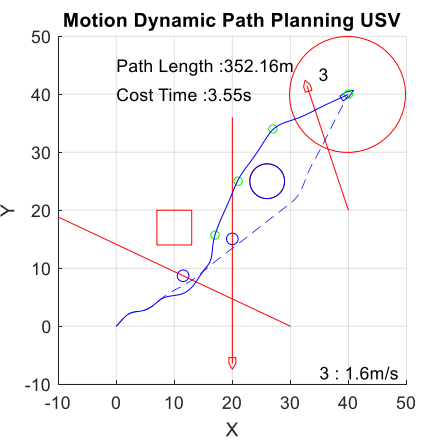

(h) $\mathrm{t}=141$ second
Fig. 12. The step of simulation in dynamic path planning

Figure 13 shows the USV trajectory during the dynamic path planning process. The blue line represents the USV trajectory generated from the trajectory tracking program (guidance system, MRAC PID controller, and real USV dynamics model). It can be seen that the USV path coincides with the target trajectory with the RMS Cross
Track Error value $=0.2437$. With the RMS Cross Track Error value for the USV trajectory in the range of 0 to 0.5 , it can be said that the trajectory tracking system (USV 6 DOF dynamic model, sliding curve guidance system, and Discrete MRAC PID) produces an accurate trajectory.

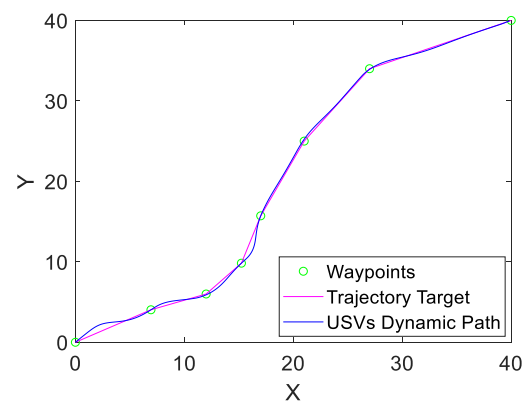

Fig. 13. Comparison of planned/ target and USV trajectory

\section{CONCLUSION}

Based on the USV trajectory tracking simulation and dynamic path planning, it can be concluded that the USV with real ship parameters is capable of navigating in a dynamic environment with external disturbances (wind, currents, and waves) without colliding static or dynamic obstacles. And also able to follow the target trajectory as a result of sliding curve guidance accurately, indicated by the RMS Cross Track Error value which is below 0.25. Discrete PID MRAC controllers are used in USV autopilot systems that have high-order and nonlinear plants to adjust the $\mathrm{X}$ axis linear velocity (surge) and the USV yaw angle. This is indicated by the test results that meet the desired design specifications and have a fairly fast settling time value and a steady-state error that is close to zero.

\section{REFERENCES}

[1] JC. Mohanta; DR. Parhi; SK. Patel, "Path planning strategy for autonomous mobile robot navigation using Petri-GA optimisation. Computers and Electrical Engineering", 2011.

[2] J. C. Latombe, "Robot motion planning," Kulwer Academic publishers, Boston, MA, 1991.

[3] T. Lozano-Perez and M. Wesley, "An algorithm for planning collision-Free paths among polyhedral obstacles", 1979.

[4] D. E. Goldberg, "Genetic algorithms in search, optimization and machine learning", Addison- Wesley publishing company, 1989.

[5] Stateczny, A.; Burdziakowski, P. Universal autonomous control and management system for multipurpose unmanned surface vessel. Polish Marit. Res. 2019

[6] Stateczny, A.; Kazimierski, W.; Gronska-Sledz, D.; Motyl, W. The Empirical Application of Automotive 3D Radar Sensor for Target Detection for an Autonomous Surface Vehicle's Navigation. Remote Sens. 2019.

[7] K. D. Do; J. Pan, "Control of Ships and Underwater Vehicles," Springer- Verlag, 2009.

[8] Y. Lu; Z. Xue; G. Xia; L. Zhang, "A Survey on Vision-based UAV Navigation", 2018.

[9] Tutorials Point n.d., the Companies Act, accessed 10 June 2020, <https://www.tutorialspoint.com/genetic_algorithms/index.

[10] Sujit, P. B., Srikanth Saripalli, and Joao Borges Sousa. "Unmanned saerial vehicle path following: A survey and analysis of algorithms for fixed-wing unmanned aerial vehicless." IEEE Control Systems Magazine 34.1 (2014): 42-59.

[11] N. Gamayanti, "Maneuver And Waypoints Control of Unmanned Surface Vehicles Using Model Reference Adaptive Control (MRAC) Based Pid Controller", 2019. 